\title{
Metodi e strategie didattiche esperienziali all'Università per incoraggiare un approccio play-based nella scuola dell'infanzia e primaria
}

\section{Experiential teaching and learning methods in Higher Education to encourage a play-based approach in pre-primary and primary school settings}

\section{Daniela Frison ${ }^{*}$}

\begin{abstract}
Riassunto
Il contributo presenta una proposta didattica ispirata ad Experiential e Active Learning rivolta a studenti e studentesse in Scienze della Formazione Primaria, con l'obiettivo di sensibilizzarli verso la centralità di un approccio play-based nella Scuola dell'Infanzia e Primaria. L'articolo presenta il framework metodologico di riferimento, gli obiettivi della strategia e la sua articolazione.
\end{abstract}

Parole chiave: peer-review, feedback tra pari, tirocinio, valutazione, auto-valutazione

\begin{abstract}
The paper presents the design process of a teaching and learning proposal addressed to students in Primary Education inspired by an Experiential and Active Learning approach. The proposed activity aims at fostering awareness about play-based learning in pre-primary and primary school settings. The paper includes the methodological framework, learning objectives and the description of the activity.
\end{abstract}

Keywords: experiential learning, active learning, play-based learning, teachers training, primary school

\footnotetext{
${ }^{*}$ Università di Firenze.

Excellence and Innovation in Learning and Teaching (ISSNe 2499-507X), 2020, 1
}

DOI: $10.3280 /$ exioa1-2020oa10077 


\section{Introduzione}

To play is to learn: è questo il titolo di uno degli interventi al World Economic Forum Annual Meeting 2018 volto a sottolineare la centralità del gioco nello sviluppo fisico e cognitivo dei bambini e delle bambine e soprattutto a denunciare la drastica riduzione dei tempi ad esso dedicati nell'extra-scuola e in famiglia (Goodwin, Polman, Brodin, \& Knell, 2018). Intorno al gioco, al gioco libero in particolare, converge l'attenzione della comunità scientifica che vede psicologi dello sviluppo, neuroscienziati, antropologi e pedagogisti concordi in merito al ruolo, chiave, che il gioco riveste nei processi di apprendimento (Whitebread et al., 2017) e nella crescita affettiva e intellettiva e altrettanto preoccupati dell'erosione dei tempi ad esso dedicati (Barblett, Knaus, \& Barratt-Pugh, 2016; Bowdon, 2015; Whitebread et al., 2017). Il tema del gioco, come evidenzia Baumgartner (2010), come un fiume carsico è apparso e scomparso dagli ambiti di studio della psicologia dello sviluppo, soprattutto dagli anni ottanta in avanti, considerato per lo più come occasione privilegiata di osservazione dello sviluppo del pensiero infantile, e non osservato quindi come attività "in quanto tale", in particolare da una prospettiva pedagogica (Bondioli, 1996). Tuttavia, il riconoscimento della centralità che le molteplici tipologie di gioco rivestono nello sviluppo delle abilità motorie, cognitive e socio-affettive di bambini e bambine e del ruolo chiave che insegnanti ed educatori possono svolgere nei contesti educativi e scolastici per promuoverlo sta tornando al centro dei tavoli di lavoro della comunità scientifica e dei policy makers (OECD, 2019).

Entro questo framework di valorizzazione del gioco, il contributo presenta una proposta didattica elaborata nell'ambito dell'insegnamento di Metodologia e tecniche del gioco e dell'animazione condotto presso l'Università di Firenze, nel Corso di Laurea in Scienze della Formazione Primaria, con l'obiettivo di sensibilizzare gli studenti e le studentesse verso la centralità di un approccio play-based nella Scuola dell'Infanzia e Primaria. La strategia sperimentata è stata progettata seguendo un approccio esperienziale all'apprendimento (Kolb, 1984; Pfeiffer, \& Jones, 1985). L'articolo presenta il framework metodologico di riferimento, gli obiettivi della strategia e la sua articolazione.

\section{L'adozione di un approccio play-based nella scuola dell'infanzia e primaria}

Il binomio gioco-apprendimento attraversa la letteratura scientifica relativa ai processi di sviluppo cognitivo di bambini e bambine (Baumgartner, 2008; Whitebread et al., 2017), allo sviluppo delle loro competenze sociali 
(Whitebread et al., 2017; Wood \& Attfield, 2005) e alla qualità dei processi di insegnamento e apprendimento (OECD, 2019). Tuttavia, le ricerche si concentrano in particolare nell' ambito dell'educazione e cura della prima infanzia (Lester \& Russell, 2010; OECD, 2019; Pramling Samuelsson, \& Johansson, 2006), mentre più limitati risultano i lavori dedicati alla centralità del gioco nei processi di insegnamento e apprendimento nella scuola primaria (Jay \& Knaus, 2018; Weisberg, Hirsh-Pasek, \& Golinkoff, 2013). Se da un lato persiste la difficoltà di giungere ad una definizione condivisa di gioco (Bondioli, 1996), dall'altro è ormai piuttosto accettata dalla comunità scientifica la definizione di play-based learning nei termini di "to learn while at play" secondo un'accezione distinta dal più ampio costrutto di gioco:

Learning is not necessary for an activity to be perceived as play but remains fundamental to the definition of play-based learning. Within studies that have examined the benefits of play-based learning, two different types of play have been the primary focus: free play, which is directed by the children themselves, and guided play, which is play that has some level of teacher guidance or involvement (Danniels \& Pyle, 2018, p. 1).

È possibile dunque collocare le attività di gioco lungo un continuum che vede da un lato il gioco libero, diretto dal bambino, guidato da una motivazione intrinseca, dalla priorità dei mezzi sul fine, dalla dominanza del soggetto sulla realtà, dalla non letteralità del gioco stesso, dalla libertà dai vincoli e, infine, dal coinvolgimento attivo (Baumgartner, 2010; Holt, et al., 2015), e dall'altro, il gioco guidato (Weisberg, Hirsh-Pasek, \& Golinkoff, 2013) inteso come "play activities with some level of adult involvement to embed or extend additional learning opportunities within the play itself" (Danniels \& Pyle, 2018, p. 1). Gli studi focalizzati sui benefici del gioco per lo sviluppo infantile hanno sottolineato, in particolare, l'importanza del gioco libero e dell'allestimento di spazitempi ad esso dedicati (Goodwin, Polman, Brodin, \& Knell, 2018; Whitebread, et al., 2017), mentre gli studi focalizzati sul ruolo del gioco nel processo di apprendimento hanno messo in evidenza la centralità del gioco guidato e la rilevanza del ruolo rivestito dall'insegnante nella progettazione e conduzione delle attività (Cutter-Mackenzie \& Edwards, 2013; Weisberg, Hirsh-Pasek, \& Golinkoff, 2013). Come evidenziano Pyle, DeLuca e Danniels (2017), limitati risultano invece gli studi che hanno affrontato il tema dell'integrazione di queste due prospettive, un'integrazione percepita come particolarmente complessa da educatori e insegnanti che segnalano la difficoltà di documentare, rendere visibile e condivisibile il valore del gioco nei processi di apprendimento, la mancanza di formazione ad un approccio play-based e la pressione, più o meno 
esplicita, ad impegnarsi verso il raggiungimento di obiettivi di apprendimento prescritti dai programmi nazionali (Howard, 2010; Leggett \& Ford, 2013).

Dagli studi a supporto di un approccio play-based nella scuola dell'infanzia e primaria, emerge un interesse a rilevare la quantità di tempo dedicata dagli insegnanti al gioco (Martlew, Stephen, \& Ellis, 2011) e ad esplorare le loro percezioni e credenze in merito al suo valore (Bennett, Wood, \& Rogers, 1997; Haney \& Bissonnette, 2011; Puteh, 2013). Interessante risulta la ricerca effettuata da Martlew, Stephen e Ellis (2011) nel contesto scozzese a partire da una traslazione dei principi guida dell'active learning e della centralità della partecipazione attiva dell'adulto nella formazione e nella didattica (Prince, 2004; Wieman, 2014) nei contesti di insegnamento rivolti ai bambini e alle bambine, a sostegno di iniziative di play-based curriculum (Scottish Executive, 2007). La ricerca mette in evidenza come gli insegnanti, seppur pienamente convinti della rilevanza del gioco nei contesti di apprendimento rivolti all'infanzia, siano incerti rispetto all'implementazione di strategie di insegnamento e apprendimento play-based. È rilevabile infatti come, nella letteratura e nelle pratiche di insegnamento focalizzate sulla scuola primaria, gioco e apprendimento risultino spesso separati (Jay \& Knaus, 2018; Pramling Samuelsson, \& Johansson, 2006) e come gli insegnanti risultino maggiormente orientati verso strategie e tecniche di apprendimento teacher-centred, fortemente orientate verso obiettivi di apprendimento specifici, incontrando difficoltà nell'articolazione di un approccio all'apprendimento play-based (Jay \& Knaus, 2018; Puteh, 2013). Come evidenziano infatti Jay e Knaus (2018):

Although play-based learning is recognised widely as a valuable pedagogical practice common to prior-to and in-school environments, it has been noted that there are often fewer opportunities for the inclusion of play-based learning activities and availability of equipment to support play in junior primary classrooms as the requirements and expectations of a more formalised curriculum begin to appear (p. 115).

Inoltre, come denunciano Fisher e colleghi (2008), i tempi destinati al gioco libero o ad attività basate sul gioco nel contesto didattico si sono via via ridotti, sempre più spesso sostituiti da lezioni frontali e strutturate, relegando il gioco ad un'attività ricreativa di secondo ordine (Pellegrini \& Bohn, 2005) da affidare all'extra-scuola e alla famiglia, soggetti educativi anch'essi tuttavia colpiti da un processo erosivo dei tempi di gioco.

Rimandando ad altre sedi una più ampia riflessione sulle misconcezioni relative al gioco e i molteplici stereotipi di cui esso soffre (Baumgartner, 2010), soprattutto in ambito scolastico, oltre che sulle categorie di gioco su cui la 
letteratura scientifica di matrice psico-pedagogica si è concentrata, dal gioco simbolico al gioco di regole, dal gioco di finzione al gioco con gli oggetti (Bondioli, 1996; Whitebread, et al., 2017), è interessante in questa sede domandarsi come, dal punto di vista didattico, il percorso universitario di formazione dei futuri e delle future insegnanti di scuola dell'infanzia e primaria possa incoraggiare l'adozione del gioco nel contesto didattico (Haney \& Bissonette, 2011) e supportare studenti e studentesse nell'intravvedere le possibili declinazioni metodologiche del gioco contribuendo al superamento dei sopra citati limiti messi in evidenza dalla letteratura.

\section{Active ed experiential learning a supporto di un approccio play- based nella scuola dell'infanzia e nella scuola primaria}

Il contributo presenta una proposta didattica elaborata nell'ambito dell'insegnamento di Metodologia e tecniche del gioco e dell'animazione condotto nel secondo semestre dell'A.A. 2018-19 presso l'Università di Firenze, Corso di Laurea in Scienze della Formazione Primaria, con l'obiettivo generale di sensibilizzare gli studenti e le studentesse verso la centralità di un approccio play-based nella scuola dell'infanzia e primaria. La strategia è stata progettata secondo due obiettivi didattici specifici:

1) allestire per gli studenti e le studentesse un'opportunità di progettazione e sperimentazione di attività di gioco pensate per i bambini e le bambine della scuola dell'infanzia e/o della scuola primaria;

2) incoraggiare, mediante un'opportuna e finalizzata attività di debriefing, una riflessione in merito alle proprie credenze sul gioco: come può essere proposto nel contesto didattico? Con quali obiettivi? Quali difficoltà intravvedo nel pensarmi, come futuro/a insegnante, conduttore/conduttrice dell'attività proposta?

A tal fine, è stata progettata un'attività definita di Animazione Tra Pari (d'ora in avanti ATP) ispirata ad un approccio esperienziale all'apprendimento (Bracci, 2017; Fedeli, Grion, \& Frison, 2016; Kolb, 1984; Pfeiffer \& Jones, 1985; Surian \& Frison, 2019) che vede il processo di apprendimento realizzarsi attraverso l'azione e la sperimentazione di situazioni in cui il soggetto è attivo protagonista e in cui viene coinvolto da un punto di vista fisico, cognitivo ed emotivo. È in particolare il learning circle tracciato da Kolb (1984), noto in particolare nell'ambito dell'apprendimento degli adulti, che ha attribuito all'apprendimento esperienziale una forma ciclica che ha inizio con un'esperienza concreta, passando per forme di osservazione riflessiva e concettualizzazione astratta, fino a giungere ad una sperimentazione attiva che nutrendo nuovamente l'esperienza concreta darà il via ad un nuovo ciclo. Gli autori che come 
Kolb si sono interessati all'apprendimento dall'esperienza - si pensi a Boud, Walker, Jarvis - si sono ispirati ai precedenti lavori di Piaget, Lewin e Dewey e hanno valorizzato il ruolo dell'esperienza stessa e della riflessione nella creazione e comprensione di idee e concetti a partire dalle osservazioni condotte (Merriam, Caffarella, \& Baumgartner, 2007).

Da un punto di vista metodologico, questo approccio ha offerto stimoli e indicazioni progettuali mirate al fine di allestire l'attività qui presentata, ispirata ai principi dell'Experiential Learning e dell'Active Learning, approccio ampiamente definito con riferimento a "anything course-related that students in a class session are called on to do other than simply watching and listening to a lecture and taking notes" (Felder \& Brent, 2016, p. 113). Attività di simulazione, sperimentazione e role-playing sono ispirate a tale approccio e richiedono ai partecipanti di agire e mettere in situazione, spesso in gruppo, nuove conoscenze e/o competenze.

Il feedback fornito nel corso della progettazione (Hattie \& Timperley, 2007) e il debriefing, inteso come processo di analisi post-esperienza (De Rossi, 2018; Fedeli, 2014) hanno inteso sollecitare la riconnessione dell'attività con gli obiettivi didattici di partenza e favorire la riflessione sulle credenze di studenti e studentesse in merito ad un approccio play-based nel contesto didattico.

\section{Animazione Tra Pari: descrizione e articolazione dell'attività}

\subsection{Organizzazione dei gruppi di lavoro e progettazione del gioco/atti- vità}

L'attività di Animazione tra Pari ha coinvolto 117 studenti e studentesse (113 F e $4 \mathrm{M}$ ) frequentanti il terzo anno del Corso di Laurea LM 85-bis in Scienze della Formazione Primaria, che hanno aderito ad essa volontariamente.

Sul versante organizzativo, gli studenti e le studentesse aderenti all'ATP si sono suddivisi in 24 gruppi da un minimo di 4 ad un massimo di 6 membri, mediante l'attività Moodle "Scelta gruppo" e ciascun gruppo è stato invitato ad individuare, progettare e proporre ad un gruppo di colleghi/e un'attività di gioco/animazione coerente con le tematiche proposte in aula. Sono state allestite nel corso dell'insegnamento (di 8 CFU per un totale di 48 ore) 12 sessioni dedicate all'ATP, della durata di 30 minuti ciascuna, che hanno previsto il coinvolgimento di 2 gruppi di lavoro. Dato l'alto numero di adesioni, i 2 gruppi coinvolti hanno negoziato e deciso quale delle 2 attività proposte (una per ciascun gruppo) mettere in azione, con il coinvolgimento di tutti i membri dei gruppi ed eventualmente di altri colleghi e colleghe. L'attività (o parte di essa) è stata realizzata a partire da una preliminare definizione, condivisa con la 
docente, focalizzata sui punti richiesti dalla scheda operativa riportata in Tabella 1 .

Tabella 1 - Scheda di descrizione del gioco/attività

\begin{tabular}{|c|c|}
\hline $\begin{array}{l}\text { TITOLO DEL } \\
\text { GIOCO/ATTIVITÀ }\end{array}$ & \\
\hline Obiettivi didattici & $\begin{array}{l}\text { Collegamento alle Indicazioni Nazionali } \\
\text { Collegamento alle Competenze Chiave e alle Competenze di } \\
\text { Cittadinanza }\end{array}$ \\
\hline Luogo di svolgimento & In aula, all'aperto, in un teatro, ecc. \\
\hline $\begin{array}{l}\text { Materiale/attrezzature } \\
\text { necessarie }\end{array}$ & $\begin{array}{l}\text { Indicare nel dettaglio i materiali e le attrezzature necessarie allo } \\
\text { svolgimento del gioco/attività }\end{array}$ \\
\hline N. partecipanti & $\begin{array}{l}\text { Indicare a che } \mathrm{n} \text {. di partecipanti si rivolge il gioco/attività (indi- } \\
\text { viduale, piccoli gruppi di n. membri, grandi gruppi, ecc.) }\end{array}$ \\
\hline Fascia d'età & $\begin{array}{l}\text { Indicare se il gioco/attività si rivolge a bambin* della scuola } \\
\text { dell'infanzia e/o primaria e specificare la fascia d'età }\end{array}$ \\
\hline Tipologia & Gioco tranquillo, moderato, attivo \\
\hline Durata & Da un min. ad un max. di... \\
\hline Svolgimento & $\begin{array}{l}\text { Descrivere dettagliatamente lo svolgimento del gioco/attività: } \\
\text { - } \text { Obiettivi } \\
\text { - } \text { Criteri da considerare per la scelta del gioco/attività } \\
\text { - } \text { Macro-progettazione } \\
\text { - } \text { Micro-progettazione } \\
\text { - } \quad \text { Ruolo e modalità di svolgimento del debriefing e modalità di } \\
\text { riconnessione con gli obiettivi di partenza } \\
\text { - } \quad \text { [Valutazione] }\end{array}$ \\
\hline \multicolumn{2}{|l|}{$\begin{array}{l}\text { Eventuali note/racco- } \\
\text { mandazioni metodolo- } \\
\text { giche }\end{array}$} \\
\hline Possibili varianti & $\begin{array}{l}\text { Possibili varianti negli obiettivi e/o nello svolgimento del } \\
\text { gioco/attività }\end{array}$ \\
\hline $\begin{array}{l}\text { Esempi di applica- } \\
\text { zione }\end{array}$ & $\begin{array}{l}\text { Con riferimento a Unità di Competenza, Unità di Apprendi- } \\
\text { mento, percorsi laboratoriali a scuola, percorsi di animazione ex- } \\
\text { tra-scolastici, ecc. }\end{array}$ \\
\hline \multicolumn{2}{|l|}{ Fonti bibliografiche } \\
\hline \multicolumn{2}{|l|}{$\begin{array}{l}\text { Riferimenti teorico- } \\
\text { metodologici }\end{array}$} \\
\hline $\begin{array}{l}\text { Autori/autrici del } \\
\text { gruppo }\end{array}$ & \\
\hline
\end{tabular}

Come riportato in Tabella 2, le 24 proposte elaborate hanno riguardato molteplici argomenti rintracciabili nella letteratura sul gioco (Baumgartner, 2010; 
Bondioli, 1996; Ferrando \& Ferràndiz, 2013; Ferranti, 2018; Resnick \& Robinson, 2017; Whitebread, et al., 2017; Staccioli, 2004, 2010) e sviluppati nel corso dell'insegnamento. Inoltre, le ATP proposte dai gruppi sono state realizzate secondo una calendarizzazione strutturata in fase di avvio dell'insegnamento e hanno consentito di sviluppare le tematiche previste dal syllabus secondo il medesimo approccio esperienziale seguito per la progettazione dell'ATP stessa. Talvolta l'ATP è stata collocata in fase di apertura e introduzione di una nuova tematica e dunque elaborata secondo una modalità flipped, fornendo precedentemente materiali e indicazioni metodologiche a supporto della progettazione (Reidsema, et al., 2017). In tal caso la partecipazione attiva all'ATP ha sollecitato un approccio induttivo al processo di insegnamento e apprendimento (Felder \& Brent, 2016). Talaltra essa è stata finalizzata a seguito della lezione focalizzata sui medesimi contenuti e ha avuto come obiettivo la sistematizzazione dei contenuti stessi e l'emersione di dubbi metodologici.

Tabella 2 - Categorie di ATP elaborate

\begin{tabular}{|l|c|}
\hline Gioco e tecnologie & 4 \\
\hline Culture in gioco & 4 \\
\hline Gioco e genere & 2 \\
\hline Gioco di regole & 2 \\
\hline Gioco e creatività & 4 \\
\hline Gioco e natura & 4 \\
\hline Gioco e narrazione & 2 \\
\hline Gioco simbolico & 2 \\
\hline & 24 \\
\hline
\end{tabular}

\subsection{Il ruolo del feedback e del debriefing}

Nella progettazione delle ATP, i processi di feedback e di debriefing hanno rivestito un ruolo chiave. I gruppi allestiti sono stati invitati a caricare la scheda con la proposta di gioco/attività in un apposito forum Moodle per gruppi separati, almeno una settimana prima della lezione in cui la simulazione era prevista, secondo il calendario distribuito in fase di avvio dell'insegnamento.

Un primo feedback è stato dunque fornito dalla docente al gruppo nel corso della fase di progettazione ed è stato focalizzato in particolare su: la coerenza tra gli obiettivi didattici da perseguire e la tipologia di gioco/attività proposta; l'adeguatezza della tipologia di gioco/attività proposta e la classe/fascia d'età identificata; e, infine, indicazioni utili alla sostenibilità della simulazione 
dell'attività stessa nel setting accademico. Obiettivo del feedback è stato evidentemente quello di fornire ai gruppi informazioni utili al miglioramento della loro progettazione e alla messa a punto della fase di simulazione offrendo agli studenti e alle studentesse stimoli mirati ad un accompagnamento verso un livello di analisi dell'attività più profondo rispetto al punto di partenza (Hattie \& Timperley, 2007; Grion \& Tino, 2018). Il feedback, infatti, in una prospettiva di assessment for learning, risulta efficace e altamente formativo, quando gli studenti hanno l'opportunità di trarne beneficio durante il percorso di apprendimento, con una ricaduta immediata sulle loro performance, superando così l'approccio dominante dell' assessment of learning (Grion \& Tino, 2018).

A seguito della simulazione, il debriefing, ossia il momento del dopo-gioco, costituisce, come evidenzia De Rossi, "un'articolata riflessione sulle azioni stesse del gioco, che ne costituisce il coronamento ai fini dell'apprendimento dall'esperienza, $[\ldots]$ una socializzazione dell'esperienza in funzione metacognitiva" (2018, pp. 156-157). Il debriefing ha consentito dunque di: raccogliere dubbi e incertezze metodologiche emerse a seguito della messa in azione dell'attività; introdurre i contenuti teorici e metodologici a partire dall'attività stessa (nel caso di un approccio flipped) o riconnetterla ad essi; far emergere le credenze di studenti e studentesse in merito al gioco, alle criticità incontrate nella progettazione, nella pianificazione e nell'esecuzione e alle sue potenzialità.

Le ricerche sui processi di feedback mettono in evidenza come i feedback fra pari siano altrettanto efficaci, se non di più, di quelli offerti dal docente (Falchikov 2005; Grion \& Tino, 2018; Nicol et al., 2014). A tale proposito il debriefing ha costituito un secondo momento di feedback offerto non solo dalla docente, ma anche da colleghi e colleghe, mirato ad orientare la finalizzazione della proposta di gioco/attività entro una settimana dalla sua sperimentazione in aula.

\subsection{La riflessione sull'azione}

Il ciclo esperienziale proposto dall'ATP ha preso dunque il via con l'attività di progettazione, seguita da un primo feedback, poi dalla finalizzazione del progetto di ATP e dalla sua simulazione riletta nella fase di debriefing e rivista alla luce di un secondo feedback fornito da docente e pari in vista della finalizzazione e consegna della proposta di ATP, come formalizzato nel ciclo di seguito proposto (Grafico 1). 


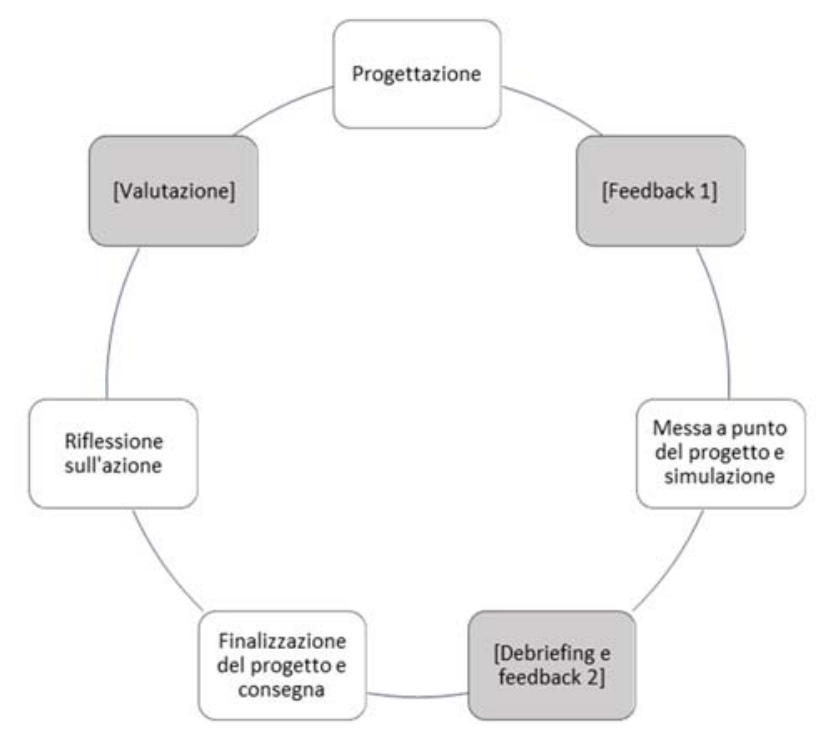

Ricordiamo qui l'obiettivo generale perseguito dalla strategia proposta ossia quello di sensibilizzare gli studenti e le studentesse verso la centralità di un approccio play-based nella scuola dell'infanzia e primaria. La riconnessione dell'attività a tale obiettivo è stata promossa mediante l'invito all'elaborazione di una riflessione personale sull'attività e in particolare sui seguenti aspetti: possibilità di connessione teoria/pratica didattica, riflessioni teorico-metodologiche sul focus dell'attività a partire dall'esperienza, punti di forza, difficoltà incontrate. L'analisi testuale dei 94 testi raccolti è in corso di elaborazione e sarà guidata dall'obiettivo di rilevare le credenze sul gioco e sull'approccio play-based nella didattica riconducibili alla fase di progettazione dell'ATP e, successivamente, collegate alla fase di simulazione, rintracciandone cambiamenti ed evoluzioni incoraggiate dalla dimensione esperienziale proposta. I risultati sosterranno sia una messa a punto della strategia di ATP dal punto di vista didattico, sia la ricerca sul play-based learning e sulle sue potenzialità nel contesto scolastico (OECD, 2019) e dei servizi per l'infanzia 0-6. Si riportano qui in ogni caso i nodi di attenzione esplicitati da studenti e studentesse, che mettono in evidenza, da un lato, le ricadute positive di un approccio esperienziale e dall'altro le criticità e le resistenze all'adozione di una prospettiva playbased.

È soprattutto la progettazione e dunque la formulazione di obiettivi didattici perseguibili mediante il gioco a sfidare i gruppi. Si riportano, a riguardo, alcuni estratti delle riflessioni che mettono in evidenza le criticità incontrate da 
studenti e studentesse nel mettere a fuoco le declinazioni metodologiche delle aree tematiche proposte.

All'inizio l'ATP ha causato dubbi e perplessità per quando riguarda la sua preparazione e realizzazione. Il tema scelto [giochi di bambine e bambini] era così ampio che è stato difficile preparare l'attività, non volevamo cadere in stereotipi o in attività già viste.

È stato un tema difficile, ma allo stesso sfidante perché, correndo il rischio di cadere in stereotipi, ci ha messo di fronte ad un tema forte e potente [culture in gioco].

È stata [...] un'occasione utile per mettersi alla prova, per sperimentare le nostre capacità e mettere in azione la nostra fantasia ed immaginazione. È stato complicato pensare in astratto a questa attività, è stata anche difficile da immaginare in un'ipotetica classe.

Emerge, tuttavia, la valenza formativa della connessione teoria-pratica percepita dai futuri e dalle future insegnanti.

Creare una connessione tra dimensione teorica e prassi è, a mio avviso, fondamentale per l'individuazione di limiti e criticità che possono derivare da un approccio di sola teoria.

Questa modalità di lavoro si basa sulla sperimentazione in prima persona delle teorie e dei metodi sui quali riflettiamo quotidianamente, ma che troppo raramente ci troviamo ad esperire durante il percorso di studi. [...] questo è senz'altro uno dei motivi che mi spingono a ritenere molto alto il valore formativo di questo tipo di attività.

Grazie a questa attività, oltre alla semplice progettazione teorica del gioco, abbiamo potuto anche rappresentarlo con l'aiuto dei nostri colleghi, così facendo siamo stati costretti a riflettere su ciò che potesse effettivamente essere messo in pratica in una classe e ciò che invece, per quanto interessante, sarebbe rimasto pura teoria. 
Poiché tutti gli output dell'ATP (schede di progettazione, materiali ad esse correlate, eventuali foto dei materiali stessi forniti dai gruppi nella fase di conduzione, ecc.) sono stati condivisi mediante uno spazio dedicato nella piattaforma Moodle, l'ATP ha reso possibile, secondo le testimonianze di studenti e studentesse, l'allestimento di un bagaglio condiviso di attività e tecniche:

Da questa esperienza imparo molto ed aggiungo al mio bagaglio professionale una serie di attività interessanti da poter proporre ai miei futuri alunni.

Per concludere, il ciclo esperienziale seguito dalla proposta di ATP ha previsto, trattandosi di un insegnamento accademico, l'attribuzione di un punteggio riconosciuto ai fini della valutazione d'esame e attribuito sulla base dei seguenti criteri di valutazione: chiarezza nella formulazione degli obiettivi di apprendimento e/o di competenza; coerenza del gioco/attività con gli obiettivi definiti; coerenza dei riferimenti teorico-metodologici con il gioco/attività proposto; completezza delle informazioni di svolgimento fornite.

\section{Conclusioni}

L'attività di Animazione Tra Pari (ATP) qui presentata e proposta agli studenti e alle studentesse del terzo anno di Scienze della Formazione Primaria, ha inteso sensibilizzare i partecipanti verso la centralità di un approccio play-based nella scuola dell'infanzia e primaria enfatizzando il ruolo chiave che l'insegnante riveste nella sua promozione attiva e mirata, in linea con la più recente letteratura internazionale focalizzata sul play-based learning. Il gioco non è ricreazione, né tempo perso, né solo un gioco: l'ATP ha consentito di sollecitare la riflessione su queste e altre misconcezioni e stereotipi che investono il gioco stesso, sia libero che guidato, portandolo ad essere distinto fattivamente dalle azioni didattiche mirate, volte ad accompagnare il processo di apprendimento e il perseguimento di obiettivi di apprendimento specifici (Jay \& Knaus, 2018; Pramling Samuelsson, \& Johansson, 2006).

La dimensione esperienziale che ha orientato la progettazione, secondo i principi dell'active learning (Prince, 2004; Wieman, 2014) e degli inductive methods (Felder \& Brent, 2016) ha messo in evidenza la centralità che il fare, la manipolazione, la sperimentazione attiva rivestono nell'attivazione di processi di critical reflection (Taylor, 2016) intorno alle proprie credenze, in qualità di (futuri e future) insegnanti, riguardo al gioco. Sono tuttavia emerse alcune criticità nel corso della realizzazione dell'ATP che sottolineano la centralità che la progettazione riveste nella proposta di metodi esperienziali. 
Primo, il numero degli studenti e delle studentesse partecipanti. Un approccio active, esperienziale, dovrebbe raggiungere la totalità dei partecipanti e garantire a tutti e a tutte pari opportunità di sperimentazione e messa in azione $\mathrm{e}$ per questo richiede un notevole investimento nella progettazione. Come evidenziano Felder e Brent (2016), infatti, una delle maggiori perplessità sollevate dai docenti nei confronti dell' Active Learning è la seguente: "I'll have to spend lots of time designing activities" (p. 126), attività che contemplino, possibilmente, il coinvolgimento dell'intero gruppo classe (nel caso specifico $117 \mathrm{stu}-$ denti e studentesse). Indubbiamente l'investimento temporale nel design delle attività è degno di nota, ma non mancano strategie e strumenti che possono supportare il docente in tale direzione: ad esempio il supporto delle tecnologie (nel caso specifico è stata adottata la piattaforma Moodle) e il lavoro in gruppi, che nel caso qui presentato si è svolto a due livelli, un primo livello di progettazione dell'attività in gruppi di 4-6 membri e un secondo livello di conduzione dell'ATP in gruppi di 10 partecipanti.

Secondo, gli spazi di apprendimento. L'ATP si è svolta in un'aula tradizionale, allestita secondo un setting frontale guidato da un modello trasmissivo del processo di apprendimento e insegnamento. Il setting è stato dunque adattato, modificato e riorganizzato per quanto possibile, per aprirsi alle attività e ai giochi proposti mettendo in evidenza come lo spazio possa costituire un limite che, se debitamente valutato in sede di progettazione, non impedisce la partecipazione attiva dell'intero gruppo classe, anche quando esso sia particolarmente numeroso.

Terzo, la gestione del feedback. Com'è noto, il feedback acquisisce centralità nel processo di apprendimento quando è tempestivo, adeguatamente progettato e strettamente ancorato ai contenuti dell'attività a cui si riferisce, fornendo agli studenti evidenze della performance da raggiungere e del gap da colmare (Hattie \& Timperley, 2007). Il passaggio dalla progettazione dell'ATP (risultata particolarmente critica per i gruppi, come evidenziato dagli estratti riportati) alla sua sperimentazione in aula è stato accompagnato da un primo feedback tempestivo, mirato e rispondente ai criteri di valutazione dell'attività. La riflessione sull'azione non ha indagato l'utilità di questo primo feedback, limite della progettazione didattica e di ricerca che potrà essere superato in occasione della prossima annualità. Tuttavia, per il momento è possibile evidenziare, nuovamente, l'investimento che il feedback, parte integrante dell'approccio esperienziale qui descritto, ha richiesto, ancora una volta gestito mediante supporto tecnologico (feedback fornito online mediante la piattaforma Moodle).

Gli elementi di progettazione didattica qui messi in evidenza intendono dunque allinearsi agli input forniti dall'agenda OECD (2019) che invita all'attivazione di percorsi di sviluppo professionale per insegnanti orientati al playful 
learning e sostiene le istituzioni scolastiche nei processi di riconoscimento del valore del gioco, valore che anche la didattica universitaria può contribuire a sostenere mediante strategie esperienziali, attive e collaborative.

\section{Bibliografia}

Barblett, L., Knaus, M., \& Barratt-Pugh, C. (2016). The pushes and pulls of pedagogy in the early years: Competing knowledges and the erosion of play-based learning. Australasian Journal of Early Childhood, 41(4), 36-43.

Baumgartner, E. (2013). Il gioco dei bambini. Roma: Carocci.

Bennett, N., Wood, E., \& Rogers, S. (1997). Teaching through play: Reception teachers' theories and practice. Buckingham: Open University Press. Google Scholar.

Biagioli, R. (2005). Educare all'interculturalità: teorie, modelli, esperienze scolastiche. Milano: FrancoAngeli.

Bondioli, A. (1996). Gioco e educazione. Milano: FrancoAngeli.

Bowdon, J. (2015). The common core's first casualty: Playful learning. Phi Delta Kappan, 96(8), 33-37.

Bracci, F. (2017). L'apprendimento adulto: metodologie didattiche ed esperienze trasformative. Milano: Unicopli.

Cutter-Mackenzie, A., \& Edwards, S. (2013). Toward a model for early childhood environmental education: Foregrounding, developing, and connecting knowledge through play-based learning. The Journal of Environmental Education, 44(3), 195-213.

De Rossi, M. (2018). Didattica dell'animazione: contesti, metodi, tecniche. Roma: Carocci.

Falchikov, N. (2005). Improving Assessment through Student Involvement. London: Routledge-Falmer.

Fedeli, M., Grion, V., \& Frison, D. (Eds.). (2016). Coinvolgere per apprendere. Metodi e tecniche partecipative per la formazione. Lecce: Pensa Multimedia.

Fedeli, M. (2014). La dimensione metodologica e riflessiva nei processi di apprendimento dall'esperienza. In M. Fedeli, L. Frontani, \& L. Mengato, (Eds.). Experiential learning. Metodi, tecniche e strumenti per il debriefing (pp. 17-26). Milano: FrancoAngeli.

Felder, R. M., \& Brent, R. (2016). Teaching and learning STEM: a practical guide. John Wiley \& Sons.

Ferrando, M., \& Ferrándiz, C. (2013). Early years' creativity. Creativity in pre-school education, 70-78.

Ferranti, C. (2018). Giocare e apprendere con le tecnologie. Esperienze da 0 a 6 anni. Roma: Carocci Faber.

Fisher, K. R., Hirsh-Pasek, K., Golinkoff, R. M., \& Gryfe, S. G. (2008). Conceptual split? Parents' and experts' perceptions of play in the 21st century. Journal of Applied Developmental Psychology, 29(4), 305-316.

Goodwin, J., Polman, P., Brodin, J., \& Knell, G. (2018). To play is to learn. Time to step back and let kids be kids. World Economic Forum Annual Meeting 2018. Retrieved online https://www.weforum.org/agenda/2018/01/to-play-is-to-learn/. 
Grion, V., \& Tino, C. (2018). Verso una "valutazione sostenibile" all'università: percezioni di efficacia dei processi di dare e ricevere feedback fra pari. Lifelong Lifewide Learning, 14(31), 38-55.

Haney, M., \& Bissonnette, V. (2011). Teachers' perceptions about the use of play to facilitate development and teach prosocial skills. Creative Education, 2(01), 41-46.

Hattie, J., \& Timperley, H. (2007). The power of feedback. Review of educational research, 77(1), 81-112.

Holt, N. L., Lee, H., Millar, C. A., \& Spence, J. C. (2015). 'Eyes on where children play': A retrospective study of active free play. Children's Geographies, 13(1), 7388.

Howard, J. (2010). Early years practitioners' perceptions of play: An exploration of theoretical understanding, planning and involvement, confidence and barriers to practice. Educational \& Child Psychology, 27(4), 91-102.

Kutnick, P., Ota, C., \& Berdondini, L. (2008). Improving the effects of group working in classrooms with young school-aged children: Facilitating attainment, interaction and classroom activity. Learning and Instruction, 18(1), 83-95.

Leggett, N., \& Ford, M. (2013). A fine balance: Understanding the roles educators and children play as intentional teachers and intentional learners within the early years learning framework. Australasian Journal of Early Childhood, 38(4), 42-50.

Lester, S., \& Russell, W. (2010). Children's right to play. An examination of the importance of play in the lives of children worldwide. Bernard van Leer Foundation. Retrieved

from http://www.academia.edu/415484/Lester S. and Russell W. 2010 Childrens ri ght to play_An_examination_of the importance_of play_in the lives_of_childr en worldwide The Hague Bernard van Leer Foundation.

Jay, J. A., \& Knaus, M. (2018). Embedding play-based learning into junior primary (Year 1 and 2) curriculum in WA. Australian Journal of Teacher Education, 43(1), 112-126.

Kolb, D. A. (1984). Experiential Learning: Experience as the source of learning and development. Englewood Cliffs, NJ: Prentice Hill.

Martlew, J., Stephen, C., \& Ellis, J. (2011). Play in the primary school classroom? The experience of teachers supporting children's learning through a new pedagogy. Early Years, 31(1), 71-83.

Munari, B. (1977). Fantasia. Roma: Laterza.

Nicol, D., Thomson, A. \& Breslin, C. (2014). Rethinking feedback practices in higher education: a peer review perspective. Assessment \& Evaluation in Higher Education, 39(1), 102-122.

OECD. (2017). Starting Strong V: Transitions from Early Childhood Education and Care to Primary Education. Online https://doi.org/10.1787/9789264276253-en.

OECD. (2019). "Play!". Trends Shaping Education Spotlights, 18. Paris: OECD Publishing. Online https://doi.org/10.1787/a4115284-en.

Pellegrini, A. D., \& Bohn, C. M. (2005). The role of recess in children's cognitive performance and school adjustment. Educational researcher, 34(1), 13-19.

Pfeiffer, W., \& Jones, J. (1985) (Eds.). A handbook of structured experience for human relations training. San Diego, US: University Associates. 
Pyle, A., DeLuca, C., \& Danniels, E. (2017). A scoping review of research on playbased pedagogies in kindergarten education. Review of Education, 5(3), 311-351.

Pramling Samuelsson, I., \& Johansson, E. (2006). Play and learning - inseparable dimensions in preschool practice. Early Child Development and Care, 176(1), 4765. Doi: 10.1080/0300443042000302654.

Prince, M. (2004). Does active learning work? A review of the research. Journal of engineering education, 93(3), 223-231.

Puteh, N. (2013). Preschool Teachers' Perceptions towards the Use of Play-Based Approach in Language and Literacy Development for Preschool. Malaysian Journal of Learning and Instruction, 10, 79-98.

Reidsema, C., Kavanagh, L., Hadgraft, R., \& Smith, N. (2017). (Eds.). The Flipped Classroom. Practice and Practices in Higher Education. Springer.

Resnick, M., \& Robinson, K. (2017). Lifelong kindergarten: Cultivating creativity through projects, passion, peers, and play. Boston, US: MIT press.

Scottish Executive. (2007). A Curriculum for Excellence, Building the Curriculum 2: Active learning in the early years. Edinburgh: Scottish Executive.

Staccioli, G. (2004). Culture in gioco: attività ludiche per l'apprendimento. Roma: Carocci Faber.

Staccioli, G. (2010). Ludobiografia: raccontare e raccontarsi con il gioco. Roma: Carocci Faber.

Surian, A., \& Frison, D. (2019). Formazione esperienziale. Proposte per la sicurezza digitale. Lecce: Pensa Multimedia.

Trinchero, R. (2012). Costruire, valutare, certificare competenze. Proposte di attività per la scuola. Milano: FrancoAngeli.

Taylor, E. W. (2016). Teacher transformation: a transformative learning perspective. Italian Journal of Educational Research, 15, 17-26.

Weisberg, D. S., Hirsh-Pasek, K., \& Golinkoff, R. M. (2013). Guided play: Where curricular goals meet playful pedagogy. Mind, Brain and Education, 7(2), 104-112.

Whitebread, D., Neale, D., Jensen, H., Liu, C., Lynneth Solis, S., Hopkins, E., HirshPasek, K., \& Zosh, J. (2017). The role of play in children's development: a review of evidence. The Lego Foundation, DK. Retrieved from https://www.legofoundation.com/media/1065/play-types-_-developmentreview web.pdf.

Wieman, C. E. (2014). Large-scale comparison of science teaching methods sends clear message. Proceedings of the National Academy of Sciences, 111(23), 8319-8320.

Wood, E., \& Attfield, J. (2005). Play, learning and the early childhood curriculum. London: Paul Chapman. 